\title{
Embarazo, parto y puerperio: creencias y prácticas de parteras en San Luis Potosí, México
}

\author{
GRAVIDEZ, PARTO E PUERPÉRIO: CRENÇAS E PRÁTICAS DE PARTEIRAS \\ EM SAN LUIS POTOSI, MÉXICO
}

\author{
PREGNANCY, DELIVERY AND PUERPERIUM: BELIEFS AND PRACTICES \\ OF MIDWIVES IN SAN LUIS POTOSI, MEXICO
}

\section{Blanca Pelcastre ${ }^{1}$, Norma Villegas², Verónica De León ${ }^{3}$, Agustín Díaz $^{4}$, Doris Ortega ${ }^{5}$, Manuel Santillana ${ }^{6}$, Juana de los Ángeles Mejía}

\section{RESUMEN}

Objetivo: Documentar las creencias y las prácticas de las parteras tradicionales respecto al embarazo parto y puerperio. Método: Aprovechando un curso de capacitación de 160 parteras tradicionales locales de las regiones Media y Huasteca del Estado de San Luis Potosí,México, dos de las enfermerasinstructoras entrevistaron a 25 de los capacitandos, apoyadas por un miembro de las comunidades náhuat y tenek. La identidad de los participantes se mantiene anónima y se obtuvo permiso para publicar los resultados mediante consentimiento informado. Resultados: Las prácticas de las parteras (os) tradicionales son comunes en los grupos sociales que carecen de servicios de salud. Sus funciones no están limitadas al parto eincluyen nutrición, cuidados prenatales, del puerperio y la lactancia, asî como apoyo afectivo emocional de las madres parturientas y sus familiares. En este estudio se registraron varios mitos y terapias tradicionales. Conclusión: Los recursos de la terapia tradicional y de la atención primaria de la salud de las parteras Náhuatly Tenek sonútiles y benéficos para los cuidados perinatales.

\section{DESCRIPTORES}

Parteras tradicionales.

Conocimientos, actitudes y

practica en salud.

Parto.

Puerperio.

Salud de las mujeres.

\section{RESUMO}

Objetivo: Documentar as cren-ças e as práticas das parteiras tradicionais quanto à gravidez, parto e puerpério. Método: Aproveitando um curso de capacitação de 160 parteiras tradicionais locais das regiões Media e Huasteca do Estado de São Luís Potosi, México, duas das enfermeiras-instrutoras entrevistaram 25 participantes do curso, apoiadas por um membro das comunidades náhuatl e tenek. Foi garantido o anonimato dos participantes, que concordaram com a publicação dos resultados, mediante o termo de consentimento esclarecido. Resultados: As práticas das (os) parteiras (os) tra-dicionais são comuns nos grupos sociais carentes de servicos de saúde. Suas funções não estão limitadas ao parto, compreendem nutrição, cuidados do pré-natal, do puerpério e da lactância, assim como apoio afetivo emocional das mães parturientes e de seus familiares. Neste estudo registraram-se vários mitos e terapias tradicionais. Conclusão: Os recursos da terapia tradicional e da atenção primaria da saúde das parteiras náhuatl e tenek são úteis e benéficas para os cuidados do pré-natal.

\section{DESCRITORES}

Parteira leiga.

Conhecimentos, atitudes e prática em saúde.

Parto.

Puerpério.

Saúde da Mulher.

\section{ABSTRACT}

Objective: To document the beliefs and practices of traditional midwifes in regard to pregnancy, delivery and puerperium. Method: Taking advantage of a training course for 160 local traditional midwives from the Media and Huasteca regions of San Luis Potosí State, Mexico. Two of the teacher-nurses interviewed 25 of them, supported by a member of Náhuatl and Tenek communities. The participant's identity was kept anonymous and permit for publications of results was obtained by informed consent. Results: Traditional midwifes practices are common in social groups lacking health services. Their role is not limited to delivery and includes nursing, prenatal and puerperium care, as well as emotional and affective support to delivering mothers and their families. In this study several myths and traditional therapies were registered. Conclusion: The traditional therapy and primary health care resources of the Náhuatl and Tenek midwifes are useful and harmless for the perinatal care.

\section{KEY WORDS}

Midwives, practical.

Health knowledge, attitudes, practice.

Parturition.

Puerperium

Women's health.
1 Doctora en Psicología Social. Jefa del Departa mento de Diseño y Modelos Alternativos de Atención. Dirección de Salud Comunitaria y Bienestar Social. Centro de Investigación en Sistemas de Salud. Instituto Nacional de Salud Pública. México. bpelcast@correo.insp.mx

2 Maestra en Salud Pública.

3 Maestra en Salud Pública.

4 Maestro en Salud Pública. Servicios de Salud del Estado de San Luis Potosí, Unidad Coordinadora Programa de Extensión de Cobertura. México

5 Maestra en Ciencias Jefa del Departamento de Equidad y Participación Social. Dirección de Salud Comunitaria y Bienestar Social. Centro de Investigación en Sistemas de Salud. Instituto Nacional de Salud Pública. México.

6 Doctor en Ciencias en Salud Pública. Instituto Mexicano del Seguro Social. Hermosillo, Sonora, México.

7 Socióloga. CIDHAL, A.C. Comunicación e Intercambio para el Desarrollo Humano en América Latina, A.C. México. 
Blanca Pelcastre

Norma Villegas

Verónica De León

Agustín Díaz

Doris Ortega

Manuel Santillana

Juana de los A. Mejía

\section{INTRODUCCIÓN: EMBARAZO Y PARTO COMO EVENTOS COLECTIVOS}

Cada cultura interpreta y da significado a su propia existencia y al mundo que la rodea, pensando y organizando la realidad de una manera determinada $^{(1)}$. Lo anterior se aplica a todos los eventos de la vida entre los que se cuenta la maternidad, que en este sentido se ve revestida de valores culturales, sociales y afectivos. Las creencias, mitos y tabúes alrededor de la maternidad muchas veces se conjugan en la figura de la partera, en quien se deposita gran parte de la responsabilidad para el cuidado de la repro-ducción biológica y sociocultural ${ }^{(2)}$.

En algunos trabajos sobre las nociones de la maternidad en poblaciones indígenas, se establece una equivalencia entre mujer y fertilidad ${ }^{(3)}$, cuya asociación prevalece en muchas regiones de México. Su estudio nos puede revelar los significados que se construyen alrededor de la condición femenina y la vivencia que se tiene de eventos como el embarazo, el parto y el puerperio. El conocimiento de estas ideas es importante, ya que de ellas se desprenden necesidades de tratamiento a base de ritos mágico-religiosos que son indispensables para el restablecimiento de la salud y cuyo manejo debe estar a cargo de personas que comparten estas mismas creencias $^{(4)}$.

La comprensión de las costumbres, las creencias y acciones asociadas a la reproducción, el embarazo, el nacimiento y la crianza de las niñas y los niños, es el primer paso para integrar esta práctica en esquemas alternativos de atención, cuyo objetivo sea la eliminación paulatina de los riesgos asociados a una maternidad en condiciones de desigualdad y marginación, y representa al mismo tiempo, la oportunidad de crear un modelo de partera, capacitada especialmente para las circunstancias de cada $\operatorname{lugar}{ }^{(5-6)}$.

\section{La experiencia subjetiva como marco referencial}

Desde una perspectiva fenomenológica, intentamos comprender este sistema de creencias, toda vez que bajo esta visión se abordan y explican los significados, que revisten de sentido las acciones cotidianas, otorgando prioridad a la experiencia subjetiva como base de este conocimiento ${ }^{(7)}$.

Por sistema de"creencias" en salud, entendemos el conjunto más o menos relacionado de valores, normas, conocimientos y comportamientos ligados a la salud, que sin ser necesariamente coherentes o estar científicamente fundados, tampoco son erróneos $^{(8)}$. En este sentido, retomamos la propuesta que sugiere que el lenguaje es un recurso para com- prender los significados que construyen realidades sociales $^{(9)}$.

\section{Las parteras tradicionales}

La Organización Mundial de la Salud (OMS) define a las parteras tradicionales como:

\begin{abstract}
...una persona (generalmente una mujer) que asiste a la madre en el curso del parto, y que inicialmente adquirió sus habilidades atendiendo ella misma sus partos o trabajando con otras parteras tradicionales; sin embargo se debe incluir, la prestación de cuidados básicos a las madres durante el ciclo normal de la maternidad, la atención del recién nacido, la distribución de métodos modernos de planificación familiar y la intervención en otras actividades de atención primaria de salud, inclusive la identificación y envío de pacientes de elevado riesgo ${ }^{(8)}$.
\end{abstract}

Las parteras tradicionales cumplen tres funciones básicas, éstas se centran en el rol que desempeñan dentro de su comunidad ${ }^{(10)}$. La primera de ellas está basada en la relación afectiva que la partera establece con las mujeres embarazadas, lo que genera confianza en estas últimas ya que la perciben cercana y familiar, esto hace que se reduzca la ansiedad en la parturienta, a diferencia de la que se provoca cuando es atendida en los servicios médicos institucionalizados. La segunda función se deriva del conocimiento que tiene la partera de las necesidades y carencias económicas de las mujeres que requieren su servicio, aunado a las propias tradiciones de la práctica, lo que conlleva a una reducción importante de costos para la usuaria. $\mathrm{La}$ tercera función se basa en el apoyo que se recibe de las parteras para las labores domésticas y el cuidado de los hijos. Estas tres funciones sumadas a la falta de otros servicios, hacen de las parteras tradicionales un recurso humano altamente demandado, sobre todo en las comunidades rurales.

En México, la participación de parteras y parteros tradicionales en la atención antes, durante y después del parto es muy importante, alrededor de un $30 \%$ de los partos son atendidos por parteras, y hasta un $100 \%$ en comunidades con mayor población indígena ${ }^{(2)}$. Acorde a las estadísticas presentadas por el Instituto Nacional de Estadística Geografía e Informática, los estados con mayor porcentaje de partos atendidos por parteras son: Chiapas (74\%), Oaxaca (56\%), Guerrero $(51 \%)$, Tabasco (42\%), Veracruz (42\%), Puebla (33\%) y San Luis Potosí $(27 \%)^{(11)}$.

En áreas rurales de alta marginalidad y difícil acceso a los servicios de salud institucionales, la figura de la partera representa el único enlace 
posible con la población materno-infantil ${ }^{(a)}$, lo que resalta aún más el apoyo potencial para las instituciones de salud.

Las parteras son el grupo de terapeutas tradicionales de mayor importancia numérica en México y el único que ha sido objeto de planes y programas institucionales de salud, al igual que en otros países de Asia y Centroamérica ${ }^{(12-14)}$, debido a que la mortalidad materna continua representando un importante problema de salud pública a pesar de los innegables avances que en esta materia ha tenido el país en los últimos años ${ }^{(b)}$.

En este contexto surgen los cursos "De partera a partera", como una iniciativa de los servicios de salud de San Luis Potosí, en la búsqueda de formas alternas y complementarias cuya diferencia con otras propuestas es la metodología de capacitación y el rescate de prácticas ancestrales de la partería tradicional, que complementa y refuerza las actividades de dependencias gubernamentales, al facilitar la integración de este valioso recurso humano a la red de Servicios de Salud ${ }^{(\mathrm{b})}$.

Un elemento fundamental que resulta imprescindible para trabajar en el marco del respeto a las creencias, es justamente el conocimiento de ellas. Este es el primer paso que permitirá tener un verdadero intercambio de conocimientos más que una capacitación unidireccional ${ }^{(6)}$. Retomando la experiencia de estos cursos y la importancia de la partería en los grupos Náhuatl y Tenek, el presente trabajo tuvo como objetivo conocer los procedimientos, prácticas y creencias con relación al embarazo, parto y puerperio, de las parteras que acuden al curso en el estado de San Luis Potosí, en el altiplano de México.

\section{MATERIAL Y MÉTODOS}

Durante la primera semana del mes de diciembre de 1998 se llevó a cabo el 3er. curso-taller De partera a partera en la ciudad de San Luis Potosí, México, como parte del Programa de Ampliación de Cobertura (PAC) de los servicios de salud de ese estado. En este curso-taller participaron alrededor de 160 parteras y parteros tradicionales de la región Media y Huasteca. Durante las sesiones de capacitación, dos enfermerasdocentes que conocían las lenguas Náhuatl y Tenek capacitadas para entrevistar, colaboraron en las actividades del curso. Se solicitó a los participantes que se sustrajeran de la capacitación por un periodo de aproximadamente 25 minutos. Las entrevistas se hicieron para conocer algunos significados de la práctica de la partería, a fin de propiciar un marco de colaboración con los servicios formales de atención al parto. Las entrevistas fueron hechas en un espacio privado, fue posible hacer cinco individuales y dos grupales; de las grupales una corresponde a miembros de la comunidad Náhuatl y otra a la Tenek. Todas las entrevistas fueron audio grabadas, previo consentimiento oral informado de los participantes y posteriormente transcritas por las enfermeras. El manejo de la información fue anónimo y confidencial. Los dos traductores que participaron en el curso apoyaron a las enfermeras durante las entrevistas y en la traducción al español y en revisión de la transcripción.

Se llevó a cabo un análisis de las conversaciones siguiendo las técnicas de la Teoría Fundamentada ${ }^{(15)}$, basada en la búsqueda sistemática de pro-piedades que permite construir una descripción más fina y detallada de la experiencia de las personas. Se revisaron todas las entrevistas por los diferentes miembros del equipo de investigación y frecuen-temente se compararon sus resultados. Se discu-tieron las diferencias y las convergencias sobre los datos entre los investigadores y se generaron nue-vas hipótesis de trabajo para las revisiones indivi-duales y grupales subsecuentes de las trans-cripciones. Se hicieron intercambios con las enfermeras entrevistadoras para clarificar el sentido de algunas palabras en español utilizadas por los entrevistados. Se utilizó la reseña del curso como material de apoyo para contextualizar las entrevistas. Se hizo la codificación con base en los con-ceptos identificados en las narraciones y se agruparon de la manera en que se presentan en el siguiente apartado.

\section{RESULTADOS}

A continuación se reportan los resultados siguiendo el esquema de los ejes temáticos utilizados para la interpretación de la información.

\section{Motivos de inicio a la partería y características de su práctica}

En esta categoría se reportan las razones por las que las parteras entrevistadas, practican la actividad de la partería, así como las condiciones en las que iniciaron y la experiencia que en ella tienen.

Este grupo de parteras y parteros se inició en esta práctica a una edad promedio de 42 años, su experiencia se ubica alrededor de los 15 años de ejercicio.

Los motivos que inician a las personas en este oficio son muy diversos, sin embargo, pueden agruparse básicamente en dos rubros: aquellas que se inician asistiendo, y en ocasiones sustituyendo
Embarazo,

parto y puerperio:

creencias y prácticas

de parteras en

San Luis Potosí,

México

$$
\begin{aligned}
& \text { (a) Información presen- } \\
& \text { tada por Soni, C., } \\
& \text { en la LII Reunión } \\
& \text { Anual de la } \\
& \text { Sociedad Mexicana } \\
& \text { de Salud Pública. } \\
& \text { A.C. Tabasco, } 17 \text { - } \\
& 21 \text { de noviembre de } \\
& 1998 . \\
& \text { (b) Reseña } 2000 \text { del } \\
& \text { Curso taller de } \\
& \text { capacitación a } \\
& \text { parteras "De } \\
& \text { partera a partera", } \\
& \text { San Luis Potosí, } \\
& \text { México. }
\end{aligned}
$$

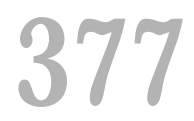

Rev Esc Enferm USP 2005; 39(4):375-82. 
Blanca Pelcastre Norma Villegas

Verónica De León

Agustín Díaz

Doris Ortega

Manuel Santillana

Juana de los A. Mejía a las parteras de edad avanzada quienes les adiestran en esta práctica; y aquellas que ante la falta de asistencia médica oportuna se vieron en la necesidad de enfrentar la atención de un parto, sin conocimiento previo, generalmente el de la esposa en el caso de los hombres.

\section{Condiciones de trabajo y recursos}

Esta categoría hace referencia a las condiciones de trabajo de las parteras, el material que utilizan, el espacio en el que atienden, la manera cómo llevan a cabo su tarea, así como las recomendaciones que hacen.

El lugar ideal para atender a la parturienta es su propia casa, sitio que ella encuentra propicio para la resolución del parto. En cuanto al espacio destinado para el parto dentro de la casa de la parturienta, encontramos el suelo como el más común. La posición preferida para el parto es muy variada, puede ser sentada, parada, acostada, hincada, o en cuclillas, dependiendo de la comodidad de la parturienta.

Respecto a la manera en que las parteras identifican que una mujer está embarazada es a través de signos tales como la palidez y el adelgazamiento y porque los ojos se le hunden. Las parteras tratan de que las mujeres embarazadas tengan un control de su estado para la adecuada atención de su parto y motivan a las mujeres a visitarlas.

Las parteras perciben que el parto está próximo generalmente guiados por la frecuencia de las contracciones:

\section{Ah, pues este, este, como diré, este, le dan más, este sí, cuando se entiesan le dan seguidito [las contracciones], y cuando ya va a nacer, le dan más seguidito, le damos unas yerbas para que le den más dolores... (E1).}

En cuanto a la higiene, limpian el lugar donde se atenderá el parto, bañan a la parturienta, le colocan un plástico y encima colocan una sábana limpia, y en el momento en el que nace el bebé cortan el cordón umbilical con carrizo o navaja, pues consideran que este tipo de material se corresponde con la cualidad "caliente"(c) de las parturientas; algunas parteras sin embargo, han incorporado el uso de tijeras respondiendo más al esquema institucional que al tradicional. Ligan el cordón con cinta o hilo, o con el mismo cordón umbilical, posteriormente bañan al bebé y a la mujer le lavan sus genitales con agua y jabón.

Proporcionan remedios para el dolor a las mujeres después del parto, le indican reposo por lo menos de 8 días y le sugieren baños. Participan también sobando (dando masajes) a las puérperas, cuidándolas y a veces preparándoles los alimentos, así como vigilando posibles complicaciones:

\begin{abstract}
Pues mire, ya cuando uno se alivia [da a luz] se le soba, se está unos días en la cama, bueno, yo creo que recostando porque hay señoras que aunque se les diga que esté acostada se levantan, yo les recomiendo la cuarentena [40 días de reposo después del parto] pues, después del parto yo les digo que no barran, ni hagan [tareas pesadas], alcen cosas pesadas, nada, porque pues muchas veces por alzar cosas pesadas o barren es cuando se les viene, se les baja la matriz, se les bajan las hemorragias... (E4).
\end{abstract}

Dentro de las complicaciones más frecuentes referidas por las parteras se cuenta la caída de la matriz, el bebé parado (posición podálica), el nacimiento de gemelos y el rompimiento de la fuente (ruptura prematura de membranas). Dificultades que no siempre pueden enfrentar de manera favorable, en algunas ocasiones con sus conocimientos los resuelven, pero cuando la situación les sobrepasa las refieren a alguna institución de salud cercana:

\begin{abstract}
Gemelos [...] este, pues ahí, el parto más [difícil], digo yo, porque a la señora se le pasaron los dolores nació uno y tenía dos horas y ya no sentía nada de dolor, de nada, ya no la atendí, no me quise hacer responsable de ello, la mandé a Zacatipán, allá la atendieron, pero ella se alivió [dio a luz] ya hasta el otro día, porque le faltaba [tiempo] a la criatura [para nacer], más por eso se le detuvieron los dolores... (E4).
\end{abstract}

El acceso a los centros de salud es un problema frecuentemente referido. Para trasladar a las mujeres embarazadas de una manera oportuna a un centro de salud u hospital, tienen que hacerlo por caminos de difícil recorrido, en algunos lugares cuentan con carreteras vecinales cuyas condiciones empeoran en tiempo de lluvia. Tienen que caminar 1 ó 2 horas y recorrer distancias mayores a 12 kilómetros.

En estas comunidades las mujeres acuden con las parteras antes que al centro de salud, además del problema anteriormente referido, esto se debe a la desconfianza que genera la institución, los altos costos, el miedo, la falta de traductores y la percepción de una práctica médica no acorde a sus costumbres:

Pues mire, como el esposo la entierra [la placenta] o sea yo nomás como quien dice yo atiendo el parto, y se le da al esposo, según él sabe lo que va hacer, enterrarla [allí] o en la esquina de la casa, pero nunca se tira, la tiran 
nada más los hospitales, porque me imagino que la han de tirar, no la entierran y siempre se entierra... (E4)

Una alimentación adecuada es básica, asegurando con ello la recuperación de la mujer después del parto y una adecuada producción de leche para el amamantamiento del recién nacido, lográndose ello a través de alimentos que se encuentran accesibles dentro de su comunidad.

\section{Creencias y mitos sobre el embarazo, el parto y el puerperio}

En este estudio se identificaron algunas creencias que revelan las preocupaciones de las mujeres y de las parteras y parteros, sobre la salud del niño y de la madre, así como el significado que se le atribuyen a ciertas costumbres durante el embarazo, el parto y el puerperio, que a continuación se describen.

El Eclipse - Efectos: la niña o el niño nace sin boca, o sin orejas, o una mano o pie; no sale bueno. No tiene dedos o nariz. El eclipse se los come de la boquita, del labio, de los dientes. Para prevenir los efectos:

\begin{abstract}
Poner en las camillas (sic.) una moneda que traiga en sus bolsillos. No salir. Ponerse un listón rojo o un seguro por el ombligo". Cuando hay eclipse y una señora está embarazada, al terminar el eclipse se lava el utensilio donde hacen las tortillas llamado comal, se junta el agua y se le da a tomar a la señora para evitar malformaciones en el producto.
\end{abstract}

El Arcoiris - Efectos: la niña o el niño sale manchado, sobre todo en la cara, para evitarlo, la partera recomienda No salir, no ver el arcoiris.

EI Mal aire tiene como efecto la enfermedad y la muerte - para evitarlo poner una cinta en la mano izquierda del recién nacido para protegerlo.

La placenta - Si se entierra correctamente los niños no se van lejos, no dejan a sus padres o las niñas no se casan lejos. Si los perros desentierran y se llevan la placenta, el recién nacido se llevará a su familia muy lejos. Si desvían la mirada cuando van a enterrar la placenta, al niño le sale la vista de lado. La persona que lleva la placenta debe ir derecho sin voltear hasta el lugar donde la van a enterrar. Se debe enterrar dentro de la casa en una esquina; la placenta y el ombligo se entierran en la esquina de la casa, donde entierran la mata de plátano o la semilla de un aguacate, las matas dan fuerza, dan fruto. Como es una vida, le prenden una vela y debe permanecer encendida. Deben entrar por la puerta con- traria a la que salieron, dar la vuelta para evitarle al niño la envidia o que lo critiquen, para que crezca sano.

Si la placenta trae dos bolitas, el próximo embarazo de la señora serán gemelar, si se deshacen se evita, pero se considera un pecado muy grande. Si la bolita es blanca será niña, si es negra será niño.

El significado del ombligo - Es carne del hijo, que no debe tirarse sino sembrarse o enterrarse; $\mathrm{Si}$ uno corta el cordón umbilical hasta que baje la placenta, ésta se enfría; Para cortar el ombligo, se debe buscar el lado caliente del ombligo.

\section{Ritos y costumbres:}

- Durante el embarazo no comer chile, jitomate y cebolla para que los niños no se hagan barrigones [les crezca el abdomen].

- Las mujeres no pueden comer frijoles después de parir, porque salen pecas en la cara, ni pescado porque dicen que se secan [dejan de producir leche].

- No comer chayotes, jitomate, ni picante después del parto porque se hinchan.

- Antes de atender un parto, la partera barre el lugar, prende una veladora y ora para que no tenga ningún problema.

- La suegra o la abuelita deben bañar al recién nacido para sentir el calor del nieto.

- [Cuando se hacen limpias con huevo], éste se rompe y si sale torcido es que tiene mal aire [enfermedad de filiación tradicional] y el niño se cura. Si el niño tiene mal aire, se tira el huevo para que se lo coman las hormigas.

- Las hierbas que se utilizan para la cura se ponen debajo del comal para que la persona que tiene mal de ojo [enfermedad de filiación tradicional] no se lo pase a otra.

- Si uno tira la hierba donde caminan otras personas, la pueden pisar y pegárseles el mal humor.

- Se tiene que pedir a Dios cuando va a nacer un niño para que no se esfume la nueva vida.

- Cobijar a la recién parturienta para que no se enfríe el estómago [la parturienta tiene cualidad caliente].

Durante los 8 días posteriores al parto, la partera encomienda al papá matar un gallito si el recién nacido fue niño y una pollita si fue niña, ambos
Embarazo,

parto y puerperio:

creencias y prácticas de parteras en San Luis Potosí, México 
Blanca Pelcastre Norma Villegas

Verónica De León

Agustín Díaz

Doris Ortega

Manuel Santillana

Juana de los A. Mejía

animales que no pesen más de un kilo; lo hacen a manera de ofrenda para evitar hemorragias y complicaciones.

La preocupación de las parteras se centra en proporcionar atención y con ello bienestar a la mujer y al bebé, esto les lleva a incorporar en su práctica remedios que por tradición y conocimiento ancestrales, ayudan a mitigar de alguna manera los malestares asociados al embarazo, el parto y el puerperio. El uso de distintos recursos denota la preocupación por aspectos como la lactancia y la anticoncepción.

En el quadro 1 (ver anexo), se resumen los recursos más ampliamente utilizados por las parteras de la región.

La motivación juega un papel importante en la actividad de todo ser humano y esto no pasa desapercibido para las parteras; la función que desempeñan dentro de sus comunidades representa el apoyo, el deber, la compañía, la ayuda, la responsabilidad, el favor a la comunidad, los vecinos y los descendientes. La comunidad reconoce y otorga a las parteras y parteros, un lugar preponderante como líderes naturales a las parteras y parteros y los gratifica de acuerdo a la confianza y posibilidades de los que demandan sus servicios:

\begin{abstract}
... hay personas que sí nos recuerdan ¿verdad? conocemos un lugar en Tének [región del estado], [donde nos dan] un bolín [semejante al pollo con unas yerbas], aparte de eso nos gratifican con un poquito de dinero [...] pues hay personas que nos dan 100 ó 200 pesos [10 ó 20 dólares], según la confianza.... (E1).
\end{abstract}

\section{DISCUSIÓN}

Es importante reconstruir eventos como el parto o el embarazo desde el punto de vista de las personas involucradas, ya que su lógica interna refleja los patrones culturales que nos permiten comprender el sentido y la importancia que guardan ${ }^{(13)}$. En este estudio hemos querido adentrarnos en las prácticas y creencias que se sustentan en algunas comunidades del estado de San Luis Potosí con relación a la partería. Esta aproximación nos ha permitido conocer y resaltar algunos aspectos cuya consideración resulta relevante en la organización de programas de capacitación dirigidos a estos recursos humanos.

Algunas de las razones por las cuales las parteras y los parteros se iniciaron en la práctica tienen que ver con la cantidad de personal médico y centros hospitalarios, que les resultan insuficientes. Cuando existen estos servicios de salud, se hace difícil o inaccesible el traslado de sus referencias, lo anterior puede ser una de las razones que ha impedido disminuir las tasas de morbi/mortalidad maternoinfantil en la región. Esta información debe tomarse en consideración para planear estrategias encaminadas a mejorar la cobertura y la accesibilidad de los servicios de salud, tanto en infraestructura como en recurso humano. La planeación debe estar orientada de acuerdo a la región y respetar las diferencias culturales de la población a la cual van dirigidas las estrategias.

La constante referencia al uso de materiales naturales utilizados para cortar el cordón umbilical, nos habla de una resistencia cultural a incorporar nuevos elementos, resistencia basada en el significado más que en la disposición. Las acciones de capacitación emprendidas desde las instituciones de salud deberían facilitar técnicas higiénicas para evitar infecciones tanto en el niño como en la madre y no tratar de sustituir el uso de estos materiales por otros. El estudio de las costumbres, creencias y prácticas debe partir del conocimiento que las sociedades crean, y de manera recíproca, dar cuenta del mundo natural y social en el que éstas han vivido $^{(6)}$

El uso de recursos disponibles se hace extensible también a la tradición alimenticia, y a los cuidados durante el embarazo y el puerperio, estos son puntos fundamentales que las parteras y las mujeres reconocen, no obstante su vigilancia depende de las condiciones de vida que tienen y que no siempre resultan favorables, lo que puede repercutir en la salud de ellas y de los recién nacidos. Este es un problema que enfatiza la necesidad de la transdiciplinariedad en salud pública, toda vez que se precisa contextualizar los problemas de salud de la población para tener una mejor comprensión de los mismos. Sin embargo, modificar las condiciones socioeco-nómicas en las que viven estas mujeres es una tarea que trasciende los objetivos de este estudio, pero desconocer estas condicionantes puede limitar el impacto esperado de toda propuesta.

Es importante resaltar el significado que tiene la placenta para las parteras y la comunidad, ya que de su manejo depende la permanencia posterior del recién nacido en la comunidad y con su familia, lo mismo puede decirse del acompañamiento o del espacio mismo donde tiene lugar el parto ${ }^{(16-17)}$, son prácticas con una gran valor cultural que revisten de significado el evento mismo del alumbramiento. Estos factores deben tomarse en cuenta en la búsqueda de estrategias institucionales que pretendan un acercamiento mayor con la población; entregar la placenta a las usuarias después de su parto, admitir un acompañante o incluso atender a la 
parturienta en su casa, son acciones que no atentan contra la salud de las mujeres y en cambio, pueden representar la posibilidad de que un mayor número de ellas confíe y haga uso de los servicios de salud.

La utilización de remedios a base de hierbas está muy extendida en estas comunidades, por su bajo costo y disponibilidad, pero más aún por el conocimiento y la experiencia que se tienen de ellas $^{(5)}$. Utilizan estos recursos para tratar padecimientos de una forma natural y no dañina, enfrentando problemas como la anticoncepción y la lactancia $^{(18)}$. Hecho importante que pone de manifiesto la necesidad de rescatar la herbolaria e incorporarla en la práctica de las instituciones de salud, como lo han señalado ya algunos auto$\operatorname{res}^{(9,19-20)}$, pues se recurre a esta práctica en la búsqueda de solución a lo que por otra parte, puede resultar incosteable.

Estas parteras y parteros forman parte de la comunidad, comparten costumbres, creencias y tradiciones, a la vez que ofrecen un servicio, razón por la cual gozan de un gran prestigio y ejercen una influencia importante sobre las prácticas de salud de la comunidad. Las instituciones ya han reco-nocido el papel de las parteras tradicionales, pero es necesario que no se deseche la posibilidad de incorporarlos como un recurso valioso en la atención primaria a la salud, por su conocimiento, experiencia, posición privilegiada y su compromiso con la población.

El reconocimiento de la importante tarea que llevan a cabo puede ser el primer paso para una relación institucional más provechosa, y para que este proceso se vea favorecido, es necesario que se produzca en un marco de sensibilización, conocimiento, difusión, seguimiento, integración y respeto basado en la interrelación sociedad-cultura-salud, que redunde en el conocimiento y respeto de las prácticas populares y procesos culturales, así como en un mejoramiento de la calidad en los servicios de salud que se ofrecen. Los Servicios de Salud no se pueden sustraer de lo que se aplica a todos los eventos de la vida y la maternidad, que no es la excepción, ya que se ve revestida de valores culturales, sociales y afectivos ${ }^{(21)}$. Cabe señalar, lo dicho anteriormente, cada cultura interpreta y da significado a su propia existencia y al mundo que la rodea, pensando y organizando la realidad de una manera determinada $^{(1)}$.
Embarazo parto y puerperio: creencias y prácticas de parteras en San Luis Potosí, México

\section{REFERENCIAS}

(1) Fairclough N. Discourse and social change. Cambridge: Polity Press; 1992.

(2) Cao L. Papel de la partería para una maternidad sin riesgos en México: ¿tradición o profesión? En: Elu MC y Santos E, editores. Una nueva mirada a la mortalidad materna en México. México: FNUAPPopulation Council; 1999. p. 195-205.

(3) Aguirre-Beltrán G. Programa de salud de la situación intercultural. México: IMSS; 1980.

(4) Tamayo R. De la magia primitiva a la medicina moderna. México: Fondo de Cultura Económica; 1997.

(5) Mellado V, Zolla C, Castañeda X. La atención al embarazo y el parto en el medio rural mexicano. México: CIESS; 1989.

(6) López-Austin A. Cuerpo humano y ideología. Las concepciones de los antiguos Nahuas. México: UNAM; 1984.

(7) Rodríguez G, Flores J y García E. Metodología de la investigación cualitativa. Granada: Aljibe; 1999. Métodos de investigación cualitativa; cap. 2; p. 39-59.

(8) Du Gas B. Parteras tradicionales. Ginebra: OMS; 1979.

(9) Berger Py Luckmann T. La construcción social de la realidad. Buenos Aires: Amorrortu; 1986.
(10) Menéndez E. Clases subalternas y el problema de la medicina denominada tradicional. México: CIESAS; 1981.

(11) Información Estadística del Sector Salud y Seguridad Social. (INEGI). Nacimientos por entidad federativa de ocurrencia según persona que atendió el parto. México: INEGI; 1999. p. 67. (Cuaderno n. 15)

(12) Castañeda X. Embarazo, parto y puerperio: conceptos y prácticas de las parteras en el estado de Morelos. Sal Pub Mex 1992; 34(5):528-32.

(13) Vargas R, Naccarato P. Allá, las antiguas abuelas eran parteras. Lima: Atenea; 1995.

(14) Zulkifli A, Rogayak Jaafar M, Hashim H, Che Wil A. Maternidad sin riesgo: una tercera alternativa para las mujeres embarazadas. Foro Mund Salud 1998; 19:136-8.

(15) Strauss A, Cordón J. Basic of qualitative research: Grounded Theory procedures and technics. Newburry Park: Sage; 1990.

(16) Davim RMB, Menezes RMP. Assistência ao parto normal no domicílio. Rev Lat Am Enferm 2001; 9(6):62-8.

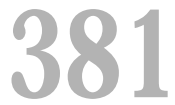

Rev Esc Enferm USP 2005; 39(4):375-82. 
Blanca Pelcastre Norma Villegas

Verónica De León

Agustín Díaz

Doris Ortega

Manuel Santillana

Juana de los A. Mejía
(17) Pinto CMS, Hoga LAK. Implantación del proyecto de inserción del acompañante en el parto: experiencias de los profesionales. Cienc Enferm 2004; 10(1):57-66.

(18) Bevilácqua DF, Neira E, Madeira LM, Laganá MT, Miranda MM. Uso popular de medicamentos de origem vegetal em lactentes. Rev Esc Enferm USP $1985 ; 19(1): 21-42$.

(19) Balcázar T, Bye R, Linares E. Remedios herbolarios usados en el centro de México. Salud Integr 2000; 1(2):34-8.
(20) Di Nicola P. Las dimensiones socioestruturales en las prácticas relativas a la salud. En: Donati P. Manual de sociología de la salud. Madrid: Diaz Santos; 1994. p. 33-47.

(21) Soares RM, Santos EM, Leal MC. Aspectos da satisfação das mulheres com a assistência ao parto: contribuição para o debate. Cad Saúde Pública 2004; 20(Supl 1):S52-S62.

\section{ANEXO}

Quadro 1 - Recursos tradicionales empleados por las/os parteras/os. (México, 2004)

Correspondência: Blanca P. Villafuerte Instituto Nacional de Salud Publica

Av. Universidad, 655

Col. Sta. Maria

Ahuacatitlán - Cuernavaca 62508 - México

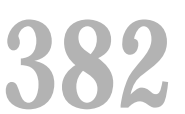

Rev Esc Enferm USP 2005; 39(4):375-82

\begin{tabular}{|c|c|}
\hline RECURSOS & usos \\
\hline $\begin{array}{l}\text { E mbarazo } \\
\text { - Tomar caldo de pollo (pollo pequeño) }\end{array}$ & Para que nazcan bien los niños \\
\hline $\begin{array}{l}\text { Parto } \\
\text { - Aceite de almendras o de avellanas } \\
\text { - Huevo batido ó hierba zimapati } \\
\text { - Té de raíz de epazote con canela y yerbabuena } \\
\text { - Hoja de naranjo ó yerbabuena ó aceite de } \\
\text { almendras untado y rebozo amarrado } \\
\text { - Endraleato (hierba de la mujer) ó cebado en aceite } \\
\text { - Aceite untado en canal vaginal } \\
\text { - Aguacate ol oroso } \\
\text { - Manzanilla (una cucharadita) } \\
\text { - Merthiolate, alcohol, tijeras, carrizo e hilo, navaja } \\
\text { de rasurar y cinta } \\
\text { - Té de epazote con cominos } \\
\text { - Dar dos palitos pequeños, pelados, machacadaos, } \\
\text { colgados en té } \\
\text { - Té de canela con manzanilla, hierbas y sobadas } \\
\text { (masajes) } \\
\text { - Té de manzanilla }\end{array}$ & $\begin{array}{l}\text { Para dar masaje en el vientre y ayudar a que } \\
\text { nazca el bebê } \\
\text { Para fortalecer a la madre durante el parto } \\
\text { Para incrementr las contracciones } \\
\text { Ayudan a acelerar el parto por su cualidad } \\
\text { "caliente". } \\
\text { Para facilitar el parto } \\
\text { Para que el bebé resbale bien } \\
\text { Para los dolores postparto } \\
\text { Para limpiar al niño recién nacido } \\
\text { Para cortar y amarrar el ombligo } \\
\text { Para detener el parto } \\
\text { Para cuando no baja la placenta } \\
\text { Para evitar hemorragias } \\
\text { Para lavar a la parturienta }\end{array}$ \\
\hline $\begin{array}{l}\text { Puerpério } \\
\text { - Ceniza de árbol ó gotas de aceite } \\
\text { - Hierba del negro con huevo y una velita } \\
\text { - Hierba verde bordoncillo, paraíso, hoja de paragos } \\
\text { - Chocolate con leche ó té de soyo (sic.) } \\
\text { - Raíz de zarza hervid ó miel de abeja tomada } 3 \\
\text { días seguidos cuando se tiene la regla } \\
\text { - Hinojo con hojitas de nuez moscada }\end{array}$ & $\begin{array}{l}\text { Para evitar que se infecte el ombligo } \\
\text { Cuando el bebé llora mucho, a los } 2-3 \text { dias de } \\
\text { nacido se le hace una limpia para espantar el mal } \\
\text { humor que le llega } \\
\text { Para hacer limpias, para que se quiten las } \\
\text { dolencias en caso de tener un mal aire } \\
\text { Para producir leche cuando no pueden amamantar } \\
\text { Para no embarazarse, seca el óvulo } \\
\text { Anticonceptivo, para que tarden en concebir } \\
\text { nuevamente } 2 \text { ó } 3 \text { años }\end{array}$ \\
\hline
\end{tabular}

\section{Agradecimientos:}

Al personal de enfermería de la Jurisdicción Sanitaria No. VI de la Unidad Coordinadora de Extensión de Cobertura de los Servicios de Salud de San Luis Potosí, que participó en la aplicación de las entrevistas y en la transcripción de las mismas. 